\title{
A new Hermite-Hadamard Type Inequality
}

\author{
Xin Chen, Yaqi Chen* and Xiang Gao \\ School of Mathematical Sciences, Ocean University of China, Lane238, Songling Road, Laoshan District, Qingdao City, \\ Shandong Province, 266100, People's Republic of China \\ ${ }^{*}$ Corresponding author
}

\begin{abstract}
In this paper, a better estimate of the Hermite type inequality for the product of two convex functions is established, and the proof is given. Then the inferences and applications of the inequalities obtained are provided.
\end{abstract}

Keywords-Hermite-Hadamard integral inequality; Convex functions

\section{INTRODUCTION}

The following definition is well-known in the literature. A real-valued function $f:[a, b] \rightarrow i, \varnothing \neq I=[a, b] \subseteq i$, is said to be convex on $[a, b]$ if inequality

$$
f(t x+(1-t) y) \leq t f(x)+(1-t) f(y)
$$

holds for all $x, y \in I$ and $t \in[0,1]$. Conversely, if the opposite inequality holds, the function is said to be concave on $I$. A function $f$ is convex on $I$ if and only if $f^{\prime \prime}(x) \geq 0$ for all $x \in(a, b)$. Let $f:[a, b] \rightarrow i$ be a convex mapping. The following inequality

$$
f\left(\frac{a+b}{2}\right) \leq \frac{1}{b-a} \int_{a}^{b} f(x) d x \leq \frac{f(a)+f(b)}{2}
$$

is known in the literature as Hadamard's inequality for convex mapping.

So far, a lot of literatures have been researched on the famous Hermite-Hadamard inequality which was first published in [1]. In recent years, some scholars have tried to improve and generalize the classical inequality. For example, ABDALLAH EL FARISSI had given new estimates in [2]; In [3], Hadamard inequality is concerned with the compound of two convex functions, which is proposed by Xiang Gao.

\section{MAIN RESULTS}

In this part, we will establish a new estimate of the product of two convex functions, which is a generalization of the following inequality:

\section{THEOREM 1.[3]}

Let $f, g:[a, b] \rightarrow[0, \infty)$ be convex functions on $[a, b] \subset i, a<b$. Then

$$
\frac{1}{b-a} \int_{a}^{b} f(x) g(x) d x \leq \frac{1}{3} M(a, b)+\frac{1}{6} N(a, b)
$$

and

$$
\begin{aligned}
& 2 f\left(\frac{a+b}{2}\right) g\left(\frac{a+b}{2}\right) \\
& \leq \frac{1}{b-a} \int_{a}^{b} f(x) g(x) d x+\frac{1}{6} M(a, b)+\frac{1}{3} N(a, b)
\end{aligned}
$$

where

$$
\begin{aligned}
& M(a, b)=f(a) g(a)+f(b) g(b) \\
& N(a, b)=f(a) g(b)+f(b) g(a) .
\end{aligned}
$$

According to the Theorem1, we can easily gain the following results.

\section{COROLLARY 1.}

Let $f, g:[a, b] \rightarrow[0,+\infty)$ be convex functions on $[a, b] \subset i$. And then meet $[f(a)-f(b)] \cdot[g(a)-g(b)] \leq 0$, we can learn that the product of $f$ and $g$ is still satisfied (1) and (2).

Secondly, we get the following main result.

(MAIN RESULT) THEOREM 2.

Let $f, g:[a, b] \rightarrow[0,+\infty)$ be convex functions on $[a, b] \subset i$, and meet $[f(a)-f(b)] \cdot[g(a)-g(b)] \leq 0$.Then for all $\lambda \in[0,1]$, we have

$$
\frac{1}{b-a} \int_{a}^{b} f(x) g(x) d x \leq L(\lambda) \leq \frac{1}{3} M(a, b)+\frac{1}{6} N(a, b)
$$

and

$$
\begin{aligned}
2 f\left(\frac{a+b}{2}\right) g\left(\frac{a+b}{2}\right)-\frac{1}{6} M & (a, b)-\frac{1}{3} N(a, b) \\
\leq l(\lambda) & \leq \frac{1}{b-a} \int_{a}^{b} f(x) g(x) d x
\end{aligned}
$$

where 


$$
\begin{aligned}
L(\lambda):=\frac{\lambda}{3} f(a) g(a)+\frac{1-\lambda}{3} f(b) g(b) \\
+\frac{1}{3} f(\lambda b+(1-\lambda) a) g(\lambda b+(1-\lambda) a) \\
+\frac{1}{6} f(\lambda b+(1-\lambda) a)[\lambda g(a)+(1-\lambda) g(b)] \\
+\frac{1}{6} g(\lambda b+(1-\lambda) a)[\lambda g(b)+(1-\lambda) g(a)]
\end{aligned}
$$

and

$$
\begin{aligned}
l(\lambda):=2 \lambda & f\left(\frac{(2-\lambda) a+\lambda b}{2}\right) g\left(\frac{(2-\lambda) a+\lambda b}{2}\right) \\
- & \frac{1+3 \lambda-3 \lambda^{2}}{6} M(a, b)-\frac{2+3 \lambda^{2}-3 \lambda}{6} N(a, b) \\
& +2(1-\lambda) f\left(\frac{(1-\lambda) a+(1+\lambda) b}{2}\right) g\left(\frac{(1-\lambda) a+(1+\lambda) b}{2}\right)
\end{aligned}
$$

\section{COROLLARY 2.}

If replace “ $[f(a)-f(b)] \cdot[g(a)-g(b)] \leq 0 \quad$ ” with neither $f$ nor $g$ are increasing or decreasing synchronously. Then for all $\lambda \in[0,1]$, the results of Theorem 2 are still satisfied.

\section{COROLLARY 3.}

Let $f, g:[a, b] \rightarrow[0, \infty)$ be convex functions on $[a, b] \subset \mathrm{i}, a<b$, and meet $[f(a)-f(b)] .[g(a)-g(b)] \leq 0$.

Then for all $\lambda \in[0,1]$, we have the following inequality

$$
\frac{1}{b-a} \int_{a}^{b} f(x) g(x) d x \leq \inf _{\lambda \in[0,1]} L(\lambda) \leq \frac{1}{3} M(a, b)+\frac{1}{6} N(a, b)
$$

and

$$
\begin{aligned}
2 f\left(\frac{a+b}{2}\right) g\left(\frac{a+b}{2}\right)-\frac{1}{6} M( & (a, b)-\frac{1}{3} N(a, b) \\
& \leq \sup _{\lambda \in[0,1]} l(\lambda) \leq \frac{1}{b-a} \int_{a}^{b} f(x) g(x) d x
\end{aligned}
$$

where $l(\lambda), L(\lambda)$ are defined in Theorem 2.

REMARK 1. Applying Theorem 2 for $\lambda=1$, we get inequality (1) and (2). In addition, if we choose $\mathrm{g}(\mathrm{x})=1$, then we have the right side of Hermite-Hadamard inequality.

\section{LEMMA}

In order to prove Theorem 2, we shall need the following Lemma:

\section{LEMMA 1.}

Let $F$ be convex functions on $[a, b] \subseteq i, a<b$, Then for all $\lambda \in[0,1]$, we have
$F\left(\frac{a+b}{2}\right) \leq \lambda F\left(\frac{\lambda b+(2-\lambda) a}{2}\right)+(1-\lambda) F\left(\frac{(1+\lambda) b+(1-\lambda) a}{2}\right)$

Proof. Let

$$
\frac{a+b}{2}=\lambda \frac{\lambda b+(2-\lambda) a}{2}+(1-\lambda) \frac{(1+\lambda) b+(1-\lambda) a}{2}
$$

Then, applying the properties of the convex function, (5) can be proved easily. W

\section{PRoOF OF The TheOREMS}

Proof of Theorem2.

Firstly, discuss about (3): Applying (1) on the subinterval $[a, \lambda b+(1-\lambda) a]$, with $\lambda \neq 0$, we have

$$
\begin{aligned}
& \frac{1}{\lambda(b-a)} \int_{a}^{\lambda b+(1-\lambda) a} f(x) g(x) d \\
& \leq \frac{1}{3}[f(a) g(a)+f(\lambda b+(1-\lambda) a) g(\lambda b+(1-\lambda) a)] \\
& \quad+\frac{1}{6}[f(a) g(\lambda b+(1-\lambda) a)+f(\lambda b+(1-\lambda) a) g(a)]
\end{aligned}
$$

Applying (1) on the subinterval $[\lambda b+(1-\lambda) a, b]$, with $\lambda \neq 1$, we have

$$
\begin{aligned}
& \frac{1}{(1-\lambda)(b-a)} \int_{\lambda b+(1-\lambda) a}^{b} f(x) g(x) d x \\
& \leq \frac{1}{3}[f(\lambda b+(1-\lambda) a) g(\lambda b+(1-\lambda) a)+f(b) g(b)] \\
& \quad+\frac{1}{6}[f(\lambda b+(1-\lambda) a) g(b)+f(b) g(\lambda b+(1-\lambda) a)]
\end{aligned}
$$

Multiplying (6) by $\lambda$, (7) by $(1-\lambda)$, then adding the inequalities, we get:

$$
\begin{aligned}
& \frac{1}{b-a} \int_{a}^{b} f(x) g(x) d x \\
& \leq \frac{\lambda}{3} f(a) g(a)+\frac{1-\lambda}{3} f(b) g(b) \\
& +\frac{1}{3}[\lambda f(b)+(1-\lambda) f(a)][\lambda g(b)+(1-\lambda) g(a)] \\
& +\frac{1}{6}[\lambda f(b)+(1-\lambda) f(a)][\lambda g(a)+(1-\lambda) g(b)] \\
& \quad+\frac{1}{6}[\lambda g(b)+(1-\lambda) g(a)][\lambda g(b)+(1-\lambda) g(a)] \\
& =\frac{1}{3}[f(a) g(a)+f(b) g(b)]+\frac{1}{6}[f(b) g(a)+f(a) g(b)]
\end{aligned}
$$


$=\frac{1}{3} M(a, b)+\frac{1}{6} N(a, b)$

So, we get that $L(\lambda)$ is defined as in Theorem 2 .

Secondly, discuss about (4):

According to (4), it also can be written as

$\frac{1}{b-a} \int_{a}^{b} f(x) g(x) d x \geq 2 f\left(\frac{a+b}{2}\right) g\left(\frac{a+b}{2}\right)-\frac{1}{6} M(a, b)-\frac{1}{3} N(a, b)$

Using the same method of above, we get

$$
\begin{aligned}
& \frac{1}{b-a} \int_{a}^{b} f(x) g(x) d x \\
& \geq 2 \lambda f\left(\frac{(2-\lambda) a+\lambda b}{2}\right) g\left(\frac{(2-\lambda) a+\lambda b}{2}\right) \\
& \quad-\frac{\lambda}{6}[f(a) g(a)+f(\lambda b+(1-\lambda) a) g(\lambda b+(1-\lambda) a)] \\
& \quad-\frac{\lambda}{3}[f(a) g(\lambda b+(1-\lambda) a)+f(\lambda b+(1-\lambda) a) g(a)] \\
& +2(1-\lambda) f\left(\frac{(1-\lambda) a+(1+\lambda) b}{2}\right) g\left(\frac{(1-\lambda) a+(1+\lambda) b}{2}\right) \\
& \quad-\frac{1-\lambda}{6}[f(\lambda b+(1-\lambda) a) g(\lambda b+(1-\lambda) a)+f(b) g(b)] \\
& \quad-\frac{1-\lambda}{3}[f(\lambda b+(1-\lambda) a) g(b)+f(b) g(\lambda b+(1-\lambda) a)] \\
& \geq 2 \lambda f\left(\frac{(2-\lambda) a+\lambda b}{2}\right) g\left(\frac{(2-\lambda) a+\lambda b}{2}\right) \\
& \quad+2(1-\lambda) f\left(\frac{(1-\lambda) a+(1+\lambda) b}{2}\right) g\left(\frac{(1-\lambda) a+(1+\lambda) b}{2}\right) \\
& \quad-\frac{1+3 \lambda-3 \lambda^{2}}{6} M(a, b)-\frac{2+3 \lambda^{2}-3 \lambda}{6} N(a, b)
\end{aligned}
$$

Let $F(x)=f(x) g(x)$, according to Lemma 1 we get

$$
\begin{aligned}
(8) \geq 2 f\left(\frac{a+b}{2}\right) g\left(\frac{a+b}{2}\right) \\
\quad-\frac{1+3 \lambda-3 \lambda^{2}}{6} M(a, b)-\frac{2+3 \lambda^{2}-3 \lambda}{6} N(a, b)
\end{aligned}
$$

$$
\begin{aligned}
L\left(\cos ^{2} \theta\right) & =\frac{\cos ^{2} \theta}{3} f(a) g(a)+\frac{1-\cos ^{2} \theta}{3} f(b) g(b) \\
+ & \frac{1}{3} f\left(b \cos ^{2} \theta+a \sin ^{2} \theta\right) g\left(b \cos ^{2} \theta+a \sin ^{2} \theta\right) \\
+ & \frac{1}{6} f\left(b \cos ^{2} \theta+a \sin ^{2} \theta\right)\left[g(a) \cos ^{2} \theta+g(b) \sin ^{2} \theta\right] \\
& +\frac{1}{6} g\left(b \cos ^{2} \theta+a \sin ^{2} \theta\right)\left[g(b) \cos ^{2} \theta+g(a) \sin ^{2} \theta\right]
\end{aligned}
$$

and

$$
\begin{aligned}
& l\left(\cos ^{2} \theta\right) \\
& =2 \cos ^{2} \theta f\left(\frac{a\left(1+\sin ^{2} \theta\right)+b \cos ^{2} \theta}{2}\right) g\left(\frac{a\left(1+\sin ^{2} \theta\right)+b \cos ^{2} \theta}{2}\right) \\
& +2 \sin ^{2} \theta f\left(\frac{a \sin ^{2} \theta+b\left(1+\cos ^{2} \theta\right)}{2}\right) g\left(\frac{a \sin ^{2} \theta+b\left(1+\cos ^{2} \theta\right)}{2}\right) \\
& -\frac{1+3 \cos ^{2} \theta-3 \cos ^{4} \theta}{6} M(a, b)-\frac{2-3 \cos ^{2} \theta+3 \cos ^{4} \theta}{6} N(a, b)
\end{aligned}
$$

\section{ACKNOWLEDGMENTS}

I would especially like to express my appreciation to my advisor professor Xiang Gao for longtime encouragement and meaningful discussions. I would also especially like to thank the referee for meaningful suggestions that led to improvement of the article.

\section{REFERENCES}

[1] J. HADAMARD, tude sur les proprits des fonctions entires et en particulier d'une fonction considre par riemann, Journal De Mathmatiques Pures Et Appliques,58(1893):171-215.

[2] ABDALLAH EL FARISSI, Simple proof and refinement of hermitehadamard inequality, Journal of Mathematical Inequalities, 4(3):365369,2010

[3] XIANG GAO, A note on the hermite-hadamard inequality, Journal of Mathematical Inequalities, 4(4):587-591, 2010.

[4] MEVLT TUN, HermiteChadamard type inequalities via $\mathrm{m}$ and $(\alpha, \mathrm{m})$ convexity., Demonstratio Mathematica, 46(3), 2013.

[5] B G PACHPATTE, On some inequalities for convex functions, Journal of Mathematical Inequalities, 3(3):315-321, 2003.

Because of $[f(a)-f(b)] .[g(a)-g(b)] \leq 0$,

$$
\text { (9) } \geq 2 f\left(\frac{a+b}{2}\right) g\left(\frac{a+b}{2}\right)-\frac{1}{6} M(a, b)-\frac{1}{3} N(a, b)
$$

Then, we get $l(\lambda)$.W

\section{EXAMPLE .}

Let $\lambda=\cos \theta^{2}, \theta \in R$, then we have 